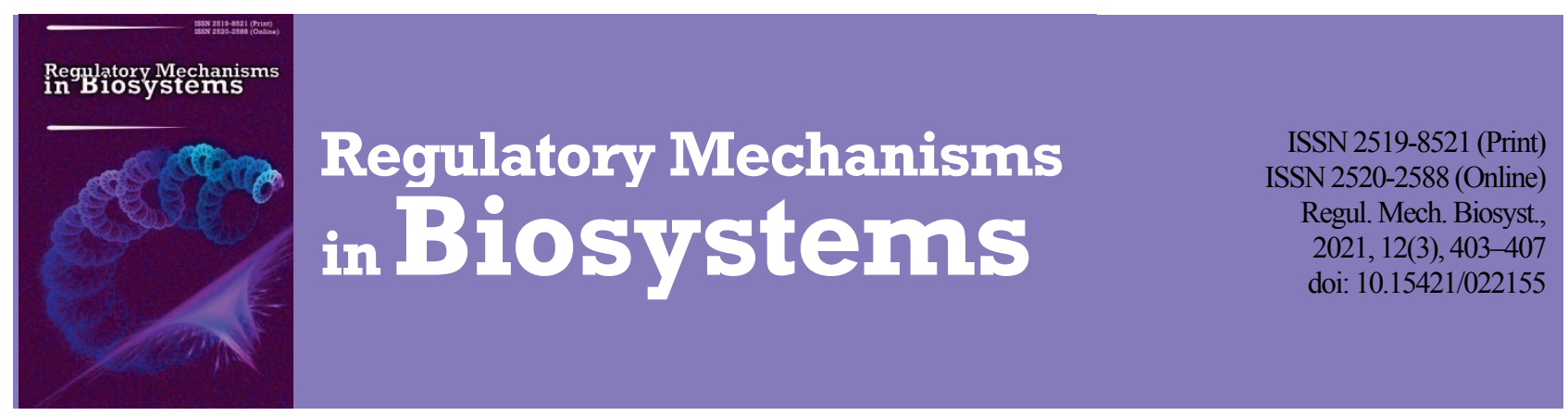

\title{
Effect of acute alcohol intoxication on scale-free neural activity in the lateral septum in rats
}

\author{
O. V. Chaikovska \\ National Pirogov Memorial Medical University, Vinnytsia, Ukraine
}

Article info

Received 02.06.2021

Received in revised form 05.07.2021

Accepted 06.07.2021

National Pirogov

Memorial Medical University

Pirogovst., 56,

Vinnytsia, 21000, Ukraine.

Tel: +38-063-267-12-13.

E-mail:

chaikovka.olga@vnmu.edu.ua

\section{Chaikovska, O. V. (2021). Effect of acute alcohol intoxication on scale-free neural activity in the lateral septum in rats. Regulatory} Mechanisms in Biosystems, 12(3), 403-407. doi:10.15421/022155

Electrophysiological recordings of brain activity show both oscillatory dynamics that typically are analyzed in the time-frequency domain to describe brain oscillatory phenomena and scale-free arrhythmic activity defined as neural noise. Recent studies consider this arrhythmic fractal dynamics of neural noise as a sensitive biomarker of a number of cognitive processes, activity of neurotransmitter systems, changes that accompany neurodegenerative and psychiatric disorders including alcohol use disorder. We tested the changes in neural noise induced by acute alcohol intoxication in the lateral septum for the entire spectrum $(1-200 \mathrm{~Hz})$ of local field potential signal and for frequency specific ranges (delta, theta, beta, gamma and epsilon bands). Five male Wistar rats were implanted with intracranial electrodes and local field potential signal was measured for baseline activity and activity induced by acute ethanol intoxication $(2 \mathrm{~g} / \mathrm{kg})$. Change in neural noise dynamics was assessed as a change in the slope of linear regression fit of power spectral density curves in double logarithmic scale. In our study alcohol resulted in lower incline of scale-free activity in the lateral septum for high frequency range and for the whole spectrum, which is interpreted generally as increase in neural noise and change in neuronal processing in a more stochastic way initiated by the acute alcohol intoxication. At the same time, we observed decrease in neural noise for low frequency range. The observed changes may be related to the shift of the excitatory-inhibitory balance towards inhibition and changes in neurotransmission mostly in the GABAergic system. Scale-free activity was sensitive in the conditions of acute alcohol intoxication, therefore to understand its role in alcohol use disorder we need more data and studies on the underlying processes. Future studies should include simultaneous recordings and analysis of arrhythmic dynamics with the oscillatory and multiunit spiking activity in the lateral septum. It can reveal the contribution of different-scale processes in changes driven by acute alcohol intoxication and clarify the specific electrophysiological mechanisms.

Keywords: neural noise; local field potential; LFP; ethanol; arrhythmic activity; neural activity; rats.

\section{Introduction}

Alcohol use disorder (AUD) is a chronic relapsing disorder characterized by non-controlled alcohol consumption with periods of abstinence and relapses. AUD imposes a heavy economic burden and is limited to therapy possibilities. Alcohol addiction is related to the complex adaptive neuroplastic changes in a number of brain areas which evolve through the consecutives stages of the cycle of abuse: stage I - acute alcohol intoxication, stage II - withdrawal/negative effect, stage III - preoccupation/craving (Koob \& Volkow, 2016). Despite the fact that many studies covered many aspects of alcohol dependence, there is still a gap in the integral understanding of the disease. This complexity of AUD is associated with involvement of multiple brain areas, neuronal circuits and neurotransmitter systems, which contribute to the disease at the different stages to various degrees. The key problem in the search of therapy for AUD is determining and unifying the potential targets for treatment.

The lateral septum (LS) is a part of the limbic and reward system which sends afferent and efferent projections to many subcortical and cortical brain areas including the ventral tegmental area, amygdala, bed nucleus of stria terminalis, nucleus accumbens, hippocampus, prefrontal cortex, etc. It is suggested that LS is involved in the formation of wide class of addictions and for instance AUD (Deng et al., 2019; Gárate-Pérez et al., 2021). In the current model of addiction, cycle the role of LS has not been determined unequivocally, implying that LS participates in formation of associations between alcohol and contextual stimuli, goalmotivated behaviour and its shift to habit that is observed in addiction, regulation of stress response, memory and emotion development. Manipulations with LS changed the course of addiction eliminating the rein- forcing action of alcohol (Jonsson et al., 2017), blocking conditional place preference for different substance of abuse (Sartor \& Aston-Jones, 2012; Gárate-Pérez et al., 2021), attenuating intake and preference of alcohol (Ryabinin et al., 2008).

Brain activity detected by the most commonly used electrophysiological techniques shows oscillatory dynamics that is typically analyzed in time-frequency domain to describe brain oscillatory phenomena (Buszaki, 2004) and broadband scale-free arrhythmic background activity, characterized as 1/f noise or pink brain noise (Freeman, 2004; Muthukumaraswamy \& Liley, 2018). Oscillatory activity is considered to emerge from correlated neuronal activity at the different scales (Buszaki, 2004; Başar, 2006; Womelsdorf et al., 2007). The neural noise is ubiquitous through the nervous system and reflects broadband generalized variability through neuronal populations (Miller \& Katz, 2010; Voytek \& Knight, 2015). $1 /$ fneural noise can be extracted from the power spectra of electroencephalogram (EEG), electrocorticogram and from local field potential (LFP) (Wen \& Liu, 2016; Molina et al., 2020). The advantages of obtaining spectral information from intracranial recordings such as LFP is the much wider frequency range (up to $400 \mathrm{~Hz}$ ) by contrast to EEG where the informative frequency range is limited to $70 \mathrm{~Hz}$ due to intense attenuation of neural activity by the soft tissues and the skull. In fact it is arrhythmic activity that forms the major part across the entire spectrum. However, there is a lack of comprehensive knowledge of the underlying processes and precise interpretation of scale-free activity, the involvement of neural noise in the processing of information, and the pathological changes are being intensely studied. The recent studies reviewed neural noise as predictors of ageing impairments in cognitive functions (Voytek et al., 2015; Dave et al., 2019) and psychiatric disorders (Molina et al., 2016; Clark et al., 2019; 
Veerakumar et al., 2019). Neural noise demonstrated sensitivity to the activity of neurotransmitter systems and drug-induced changes, including alcohol (Petermann et al., 2019; Molina et al., 2020; Stock et al., 2020). In this study, we focused on how alcohol changes the scale-free dynamics of neural noise extracted from the local field potential spectrum in the lateral septum for different frequency bands in range $1-200 \mathrm{~Hz}$.

\section{Materials and methods}

The study was approved by the Ethics Committee of National Pirogov Memorial Medical University, Vinnytsya, Ukraine. All animal experiments were conducted according to the recommendations of "Guide to the care and use of laboratory animals" (8th edition, National Academy Press, Washington, 2010) and the EU Directive 2010/63/EU for care and use of laboratory animals and with the European Convention for the Protection of Vertebrate Animals Used for Scientific Purposes (Strasbourg, 1986). Eight male Wistar rats were used for the experiment ( $\mathrm{m}=170$ $250 \mathrm{~g} ; 3$ months, Bogomoletz Institute of Physiology of National Academy of Science, Kyiv, Ukraine). The rats were housed in cages (one rat per cage) for adaptation under controlled light, temperature and humidity conditions with free access to water and food.

Surgery and electrode implantation: combination of ketamine $(90 \mathrm{mg} / \mathrm{kg}$ ) and xylazine (12 mg/kg) in intraperitoneal (ip) injections were used for anesthesia. Rats were placed in the stereotaxic instrument for surgery on the heating pad to maintain constant temperature during the surgery and experiment. A skull hole was drilled in the accordance with the coordinates of LS: AP $+0.53 \mathrm{~mm}, \mathrm{ML} \pm 0.8 \mathrm{~mm}$, DL $-5.2 \mathrm{~mm}$ (Paxinos \& Watson, 2006) and microelectrode array was implanted into LS (with micromanipulator step $5 \mu \mathrm{m}$ ). Location was confirmed by electrolytic lesions with direct current $(5 \mathrm{~mA}$, duration $12 \mathrm{~s})$.

LFP recordings and data analysis: LFP of spontaneous activity was recorded with 8-channel tungsten electrode 10 minutes for basic activity and 20 minutes for period of acute alcohol intoxication. Acute intoxication was modelled by ip injection of $15 \%$ solution of ethanol $(2 \mathrm{~g} / \mathrm{kg})$. LFP was recorded with the data acquisition module (Chaikovska et al., 2019). The signal was digitalized with the sampling rate 20,880 samples/channel. Offline signal processing was performed in Matlab, Mathworks. The raw signal was digitally filtered with 5 th order low pass Butterworth filter with cutoff frequency of $400 \mathrm{~Hz}$ and downsampled to the 1,305 samples/channel. Segments with duration of 5 minutes for baseline activity and acute intoxication were used for analysis.

Typically Welch power spectral density (PSD) estimation method was used to analyze spectral characteristics of LFP signal and powers of frequency specific bands. Analysis of scale free dynamics can also be assessed from spectrum. Neural noise has power law or 1/f $\beta$ behaviour. Parameter $\beta$ is used as estimate of neural noise behaviour. Linear fit of PSD (see Equation 1) in double logarithmic coordinates gives an estimation of frequency scaling exponent by the slope of the line $\beta$, where $\mathrm{P}$ is the PSD of scale free part of spectrum, $f$ is the frequency, $\alpha$ is intercept and $\beta$ is the slope of the line:

$$
\log (\mathrm{P})=\alpha-\beta \log ; 0)(\mathrm{f})
$$

However, PSD obtained with Welch technique contains both oscillatory activity (Buzsáki \& Draguhn, 2004) and the scale free or fractal dynamics (Miller et al., 2009; Stock et al., 2020). Important oscillations affect the power distribution and behaviour of linear fit (Dave et al., 2018). Several techniques were used to split oscillations and scale-free parts of the spectra (Yamamoto \& Hughson, 1993; Wen \& Liu, 2015).

To separate scale free activity from oscillations we used Irregularly Resampled Auto Spectral Analysis (IRASA) as introduced in (Wen \& Liu, 2016). IRASA was performed in Matlab (code was presented by Wen \& Liu (2015)) for accurate evaluation of the arrhythmic activity. IRASA used the following algorithm to separate the scale free part from the general spectrum: the analyzed data were divided in 15 snippets, then the fast Fourier transform (FFT) was calculated for each snippet. All power spectra are averaged to obtain the mixed power spectra of the signal. The snippets then were resampled with resampling factors $h$ and $1 / h$, where $h$ varied 1.1 to 1.9 with the step of 0.05 . Then the power spectra were calculated for resampled snippets of data using the same FFT method. The geometric mean values of power spectra were calculated for each snippet for each $\mathrm{h}$ and its reciprocal $1 / \mathrm{h}$. The scale-free part of the spectrum was evaluated as a median for all values of $h$.

Linear regression was applied in Matlab (with the function robustfit) to calculate the slope $\beta$ of fitted line for scale-free PSD in log-log scale (Eq. 1). The detailed graphic chart of signal processing stages to obtain linear fit is presented in Figure 1.

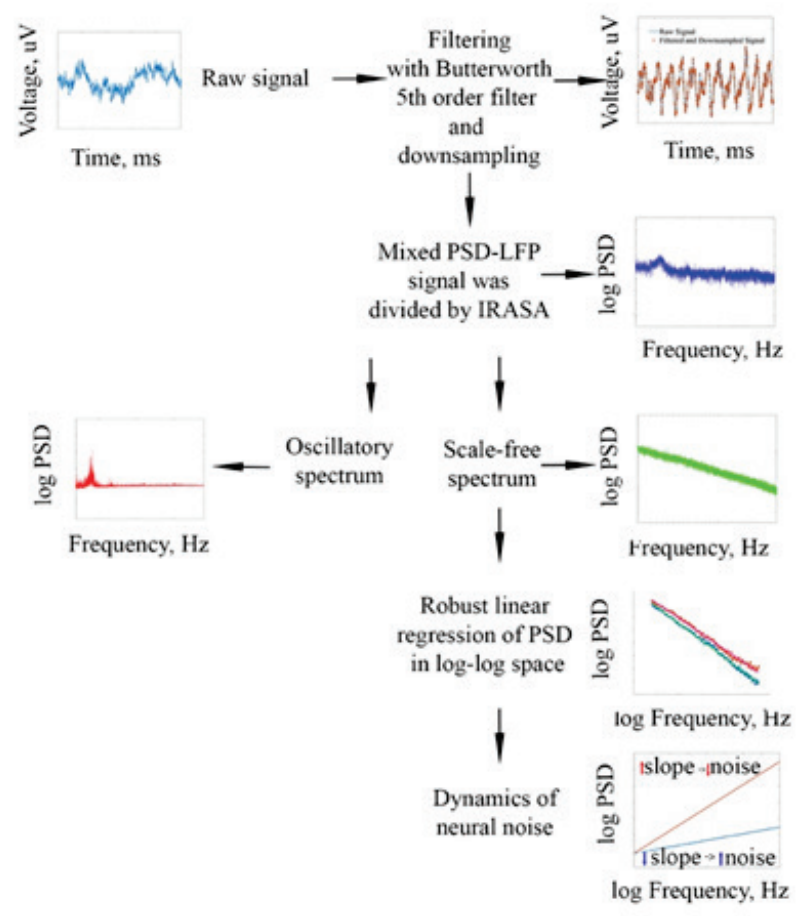

Fig. 1. Flowchart of the data processing stages to assess dynamics of neural noise by the slope of the linear regression fit $\beta$ of scale-free part of PSD-LFP signal: PSD - power spectral density, LFP - local field potential, IRASA - Irregularly Resampled AutoSpectral Analysis, $\mathrm{SD}$ - standard deviation

The values for parameter $\beta$ for baseline activity and acute intoxication obtained by linear fit were presented as median \pm standard deviation (SD) and compared with Mann-Whitney U test (P-value threshold $<0.05$ was determined as statistically significant). Coefficient of determination for linear fit was calculated as $\mathrm{R}^{2}=1-\mathrm{SSE} / \mathrm{SST}$, where SSE is the sum of squared error, SST is the sum of squared total. Statistical analysis was performed in Matlab, Mathworks.

\section{Results}

The linear regression fit of PSD-LFP was calculated for delta (1$4 \mathrm{~Hz})$, theta $(4-12 \mathrm{~Hz})$, beta $(12-30 \mathrm{~Hz})$, gamma $(30-90 \mathrm{~Hz})$ and epsilon $(90-200 \mathrm{~Hz}$ ) frequency bands and is shown in Figure 2a-e and for whole spectrum in range 1-200 Hz in Figure 2f using robust regression method.

Equations of linear fits of PSD-LFP signal in LS with intercepts and slopes for all spectrum and frequency specific bands were summarized in Table 1. Slopes of the linear fit were extracted to assess the dynamics of neural noise (Table 2). Slope for delta range (Fig. 2a) increased after acute alcohol intoxication from $-2.74 \pm 0.24$ to $-3.53 \pm 0.40(\mathrm{P}<0.01)$. Slope for theta range (Fig. 2b) of baseline activity was $-2.25 \pm 0.53$ and under action of ethanol increased to $-2.34 \pm 0.57(\mathrm{P}>0.05)$. The tendency remained the same for beta band (Fig. 2c) and increase in slope was detected from $-2.61 \pm 0.30$ to $-2.79 \pm 0.31(\mathrm{P}<0.05)$. The estimated slopes for the gamma frequency band (Fig. 2d) were $-2.62 \pm 0.32$ for baseline PSD and $-2.37 \pm 0.27$ after ethanol injection $(\mathrm{P}<0.05)$. Analysis of linear fit of the epsilon band (Fig. 2e) revealed the change in regression parameters $-2.33 \pm$ 0.26 compared with $-1.47 \pm 0.10(\mathrm{P}<0.01)$ under action of ethanol. Slope for the whole spectrum (Fig. 2f) tended to flattening with the parameter of regression $-2.57 \pm 0.30$ for baseline activity against $-2.15 \pm 0.23$ for acute intoxication $(\mathrm{P}<0.05)$. 

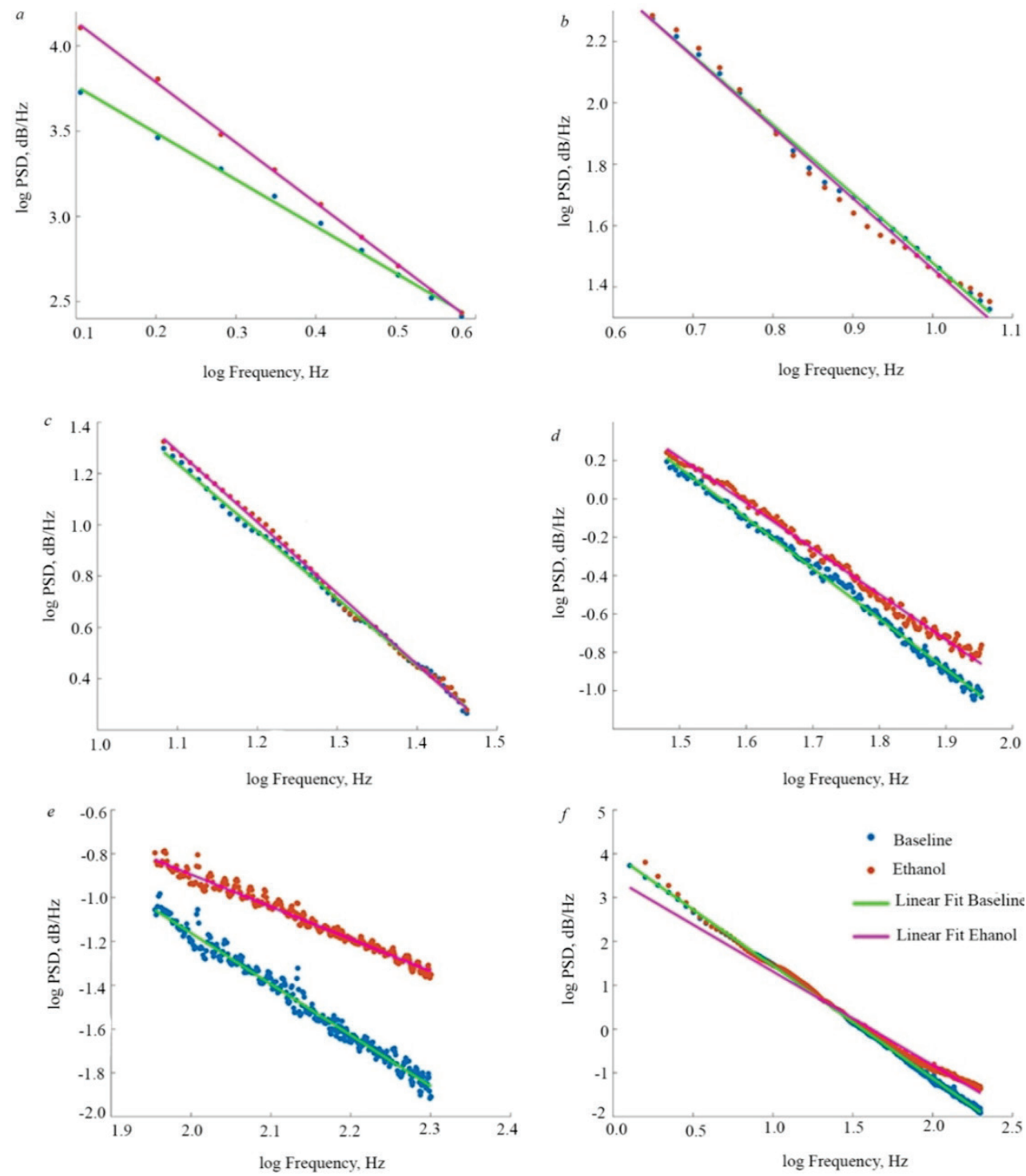

Fig. 2. Linear fit for scale-free LFP-PSD of lateral septum for delta frequency range (A), theta frequency range (B), beta frequency range (C), gamma frequency band (D), ultrafast (epsilon) frequency band (E) and for all spectrum (F); blue dots - baseline activity, red dots - activity during acute alcohol intoxication, green line - linear fit for baseline activity, pink line - linear fit for activity during acute alcohol intoxication;

PSD - power spectral density, EtOH - ethanol intoxication; $n=8$

Table 1

Linear fit equation for PSD-LFP signal in lateral septum $\log _{10}(\mathrm{PSD})=$ intercept + slope* $(\log (\mathrm{f})) /$ R-squared for linear fit

\begin{tabular}{lcc}
\hline \multicolumn{1}{c}{$\begin{array}{c}\text { Frequency } \\
\text { range }\end{array}$} & $\begin{array}{c}\text { Linear fit } \\
\text { for baseline activity } / \mathrm{R}^{2}\end{array}$ & $\begin{array}{c}\text { Linear fit for acute } \\
\text { alcohol intoxication } / \mathrm{R}^{2}\end{array}$ \\
\hline Delta, $1-4 \mathrm{~Hz}$ & $4.03-2.74 * \log (\mathrm{f}), \mathrm{R}^{2}=0.99$ & $4.49-3.53 * \log (\mathrm{f}), \mathrm{R}^{2}=0.99$ \\
Theta, 4-12 Hz & $3.73-2.25 * \log (\mathrm{f}), \mathrm{R}^{2}=0.89$ & $3.76-2.34 * \log (\mathrm{f}), \mathrm{R}^{2}=0.88$ \\
Beta, $12-30 \mathrm{~Hz}$ & $4.12-2.61 * \log (\mathrm{f}), \mathrm{R}^{2}=0.96$ & $4.34-2.79 * \log (\mathrm{f}), \mathrm{R}^{2}=0.99$ \\
Gamma, $30-90 \mathrm{~Hz}$ & $4.10-2.62 * \log (\mathrm{f}), \mathrm{R}^{2}=0.99$ & $3.77-2.37 * \log (\mathrm{f}), \mathrm{R}^{2}=0.99$ \\
Epsilon, $90-200 \mathrm{~Hz}$ & $3.49-2.33 * \log (\mathrm{f}), \mathrm{R}^{2}=0.98$ & $2.05-1.47 * \log (\mathrm{f}), \mathrm{R}^{2}=0.97$ \\
All range, $1-200 \mathrm{~Hz}$ & $4.01-2.57 * \log (\mathrm{f}), \mathrm{R}^{2}=0.99$ & $3.45-2.15 * \log (\mathrm{f}), \mathrm{R}^{2}=0.98$ \\
\hline
\end{tabular}

Table 2

Change in slope $\beta$ of linear regression fit of PSD-LFP signal in lateral septum for frequency specific bands (median $\pm \mathrm{SD} ; \mathrm{n}=8$ )

\begin{tabular}{lccc}
\hline \multicolumn{1}{c}{$\begin{array}{c}\text { Frequency } \\
\text { range }\end{array}$} & $\begin{array}{c}\text { Slope for PSD } \\
\text { (baseline activity) }\end{array}$ & $\begin{array}{c}\text { Slope for PSD } \\
\text { (acute intoxication) }\end{array}$ & $\begin{array}{c}\text { Signifi- } \\
\text { cance }\end{array}$ \\
\hline Delta, 1-4 Hz & $-2.74 \pm 0.24$ & $-3.53 \pm 0.40$ & $\mathrm{P}<0.01$ \\
Theta, 4-12 Hz & $-2.25 \pm 0.53$ & $-2.34 \pm 0.57$ & $\mathrm{P}>0.05$ \\
Beta, $12-30 \mathrm{~Hz}$ & $-2.61 \pm 0.30$ & $-2.79 \pm 0.31$ & $\mathrm{P}<0.05$ \\
Gamma, 30-90 Hz & $-2.62 \pm 0.32$ & $-2.37 \pm 0.27$ & $\mathrm{P}<0.05$ \\
Epsilon (ultrafast), 90-200 Hz & $-2.33 \pm 0.26$ & $-1.47 \pm 0.10$ & $\mathrm{P}<0.01$ \\
All range, 1-200 Hz & $-2.57 \pm 0.31$ & $-2.15 \pm 0.23$ & $\mathrm{P}<0.05$ \\
\hline
\end{tabular}

Values for parameter $\beta$ which reflect the behaviour and dynamics of neural noise for baseline activity and change after the acute alcohol intoxication for all frequency ranges are summarized in Figure 3.

Our results revealed that neural noise decreased in the slope of linear fit in low frequency range for delta, theta and beta bands and increased in high frequency range for gamma and epsilon bands under the action of ethanol. As for the behaviour of the entire spectrum, there was flatter incline for acute alcohol intoxication.

\section{Discussion}

Scale-free arrhythmic activity or neural noise are considered to represent different aspects of underlying processes in the brain, such as natural variability responses of neurons to stimuli (Dinstein et al., 2015), balance of excitation-inhibition, activity of specific neurotransmitter systems (Pertermann et al., 2019), adaptive gating mechanism to stabilize the response to stimuli (Daunizeau et al., 2012). The previously prevalent point of view where increase in noise leads to decrease in quality of communication, as claimed by Shannon (1948), was expanded by several modelling studies supported by the experimental data suggesting that neural noise is beneficial for non-linear systems and can facilitate processing of information by complex neuronal networks (Kosko \& Mitaim, 2003; Miller \& Katz, 2010; McDonnell \& Ward, 2011). Neural noise up to some threshold can 
help to perceive weak stimuli in the threshold-dependent systems, when weak signals would not reach detection level in noise-free conditions (Kosko \& Mitaim, 2003; González-Villar et al., 2017). In addition to classical approaches based on brain oscillations analysis, the neural noise provides complementary information about neural dynamics. Thus, it shows perspective as a sensitive biomarker to detect changes in neural processes (Voytek et al., 2015; González-Villar et al., 2017; Clark et al., 2019; Dave et al., 2019).

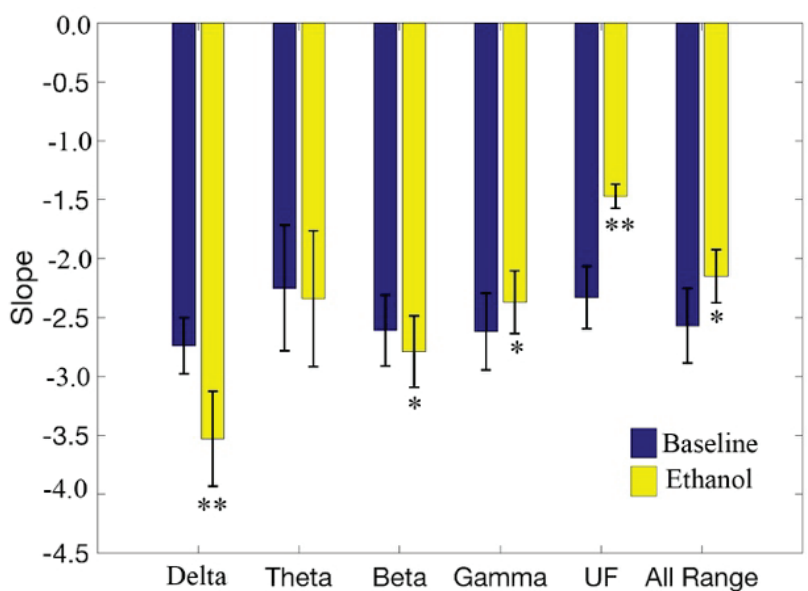

Fig. 3. Change in the dynamics of neural noise assessed from the slope $\beta$ of linear fit for PSD-LFP in the lateral septum: baseline activity (blue bars) compared with acute alcohol intoxication (yellow bars);

${ }^{*}-\mathrm{P}<0.05$, ** $-\mathrm{P}<0.01$; UF (epsilon) - ultrafast range, $\mathrm{PSD}$ - power spectral density, LFP - local field potential; EtOH - acute ethanol intoxication; median $\pm \mathrm{SD}, \mathrm{n}=8$

Acute alcohol intoxication changed LFP profile in LS for all frequency bands estimated in frequency domain by power analysis, and the most significant changes in power were detected for delta, gamma and epsilon frequency ranges (Porjesz \& Begleiter, 2003; Rangaswamy \& Porjesz, 2014). Delta band is reviewed as responsible for slow coordination between distant brain areas, beta and gamma frequency ranges were considered as reflection of ensemble activity of local neural networks (Buszaki, 2006; Buszaki \& Anastassiou, 2011). Ultrafast oscillations (epsilon band) refer to the patterns of cell activations (Schomburg et al., 2012).

Approximation of the PSD slope with linear fit in double logarithmic coordinates, proposed by Voytek (2015) with colleagues, gave an estimation of neural noise. Flattened PSD curve is interpreted as increase of neural noise and steeper slope means decrease in noise. In this study we observed that alcohol intoxication caused decrease of neural noise or steepened slope of PSD for low frequencies with increase in noise for high frequency range.

Increased neural noise for high frequency range likely leads to attenuation of signal-noise ratio in neural networks under the action of alcohol (Pertermann et al., 2019) by disorganization of oscillatory synchrony in neural networks (Voytek et al., 2015). Decrease in neural noise for betagamma ranges may be interpreted as changes in GABA activity and increased inhibition (Gao et al., 2017). The similar effect of alcohol on neural noise was described in (Stock et al., 2019) for beta band in human volunteers as decrease in $1 / \mathrm{f}$ neural noise in the state of rest for EEG recordings during binge drinking and was attributed to activation of GABAergic system under the influence of alcohol. This frequency range is specifically sensitive for inhibition driven by GABAA neurotransmission (Baumgarten et al., 2016; Osinski et al., 2018; Stock et al., 2019). Decreased neural noise was observed also for schizophrenia patients and in the opinion of the authors the slope of PSD linear fit can be a predictor of schizophrenia and the reduced and disorganized neural communications during this disease may be explained in this framework (Molina et al., 2016). Other studies revealed increase in neural noise dynamics in fibromyalgia cognitive impairment (González-Villar et al., 2017) and in agerelated changes (Voytek et al., 2015) and these effects were connected both with the impairment of excitatory-inhibitory ratio and firing correlations in activity of neurons. Our results may suggest that high doses of al- cohol tend to change the dynamics of neural noise in an opposite way for low and high frequencies as reflection of different underlying processes: change in inhibitory-excitatory balance, which can be explained by the shift to inhibition at least through enhancing of GABA signaling and increasing in dopaminergic neurotransmission induced by alcohol (Gao et al., 2017; Stock et al., 2020), and increase in the variability for individual neuronal activity and increase in randomness at population level (Ehlers et al., 2012) for high frequency range.

Our findings and data from different research groups confirmed that arrhythmic activity or neural noise changes at the first stage of the addiction cycle, acute alcohol intoxication. Similar effects were observed in numbers of other diseases and conditions that involve changes in neural activity and neurotransmission along with the impairment in oscillatory activity. More data and models revealing the background processes behind the neural noise dynamics are needed to substantially explain these changes.

\section{Conclusions}

Acute alcohol intoxication had the evident influence on the dynamics of neural noise in LS. Our findings revealed that alcohol flattened the scale free $1 /$ f signal in LS for gamma and epsilon bands, which is interpreted as increase in neural noise. The opposite effect was observed for delta, theta and beta frequency ranges. Low frequency bands exhibited the decrease in neural noise. The whole spectrum dynamics tended to flatten under the action of alcohol and showed increase in arrhythmic activity. As was reported previously, alcohol alters the nonlinear structure of brain activity and disorganizes the correlated activity in neuronal pools which was manifested as increase in neural noise, which is consistent with the behaviour for the whole spectrum and high frequency range. Decrease in noise for low frequency range can be related to the shift to inhibition in excitatoryinhibitory balance, which was the greatest overall effect of ethanol on the brain activity mediated by GABA transmission, as a possible effect of dopaminergic modulation that had contributed to the decrease of arhythmical activity.

The authors declare that they have no conflict of interests.

This study did not use any external funding.

\section{References}

Başar, E. (2006). The theory of the whole-brain-work. International Journal of Psychophysiology, 60(2), 133-138.

Baumgarten, T. J., Oeltzschner, G., Hoogenboom, N., Wittsack, H. J., Schnitzler, A., \& Lange, J. (2016). Beta peak frequencies at rest correlate with endogenous $\mathrm{GABA}+/ \mathrm{Cr}$ concentrations in sensorimotor cortex areas. PloS One, 11(6), e0156829.

Buzsaki, G. (2006). Rhythms of the brain. Oxford University Press, New York.

Buzsáki, G., \& Draguhn, A. (2004). Neuronal oscillations in cortical networks. Science, 304(5679), 1926-1929.

Buzsáki, G., Anastassiou, C. A., \& Koch, C. (2012). The origin of extracellular fields and currents - EEG, ECoG, LFP and spikes. Nature Reviews Neuroscience, 13(6), 407-420.

Chaikovska, O., Ponomarenko, O., Dovgan, O., Rokunets, I., Pavlov, S., Kryvoviaz, O., \& Vlasenko, O. (2019). Concept and realization of backpack-type system for multichannel electrophysiology in freely behaving rodents. Informatics Control Measurement in Economy and Environmental Protection, 9(4), 64-68.

Clark, D., Brown, E., Skarsgard, M., Ramasubbu, R., \& Kiss, Z. (2019). 1/f neural noise predicts outcome to subcallosal cingulate deep brain stimulation for treatment-resistant depression. Biological Psychiatry, 85(10), S350-S351.

Daunizeau, J., Stephan, K. E., \& Friston, K. J. (2012). Stochastic dynamic causal modelling of fMRI data: Should we care about neural noise? NeuroImage, 62(1), 464481

Dave, S., Brothers, T. A., \& Swaab, T. Y. (2018). 1/f neural noise and electrophysiological indices of contextual prediction in aging. Brain Research, 1691, 3443.

Deng, K., Yang, L., Xie, J., Tang, H., Wu, G. S., \& Luo, H. R. (2019). Whole-brain mapping of projection from mouse lateral septal nucleus. Biology Open, 8(7), bio043554.

Ehlers, C. L., Wills, D. N., \& Havstad, J. (2012). Ethanol reduces the phase locking of neural activity in human and rodent brain. Brain Research, 1450, 67-79. 
Freeman, W. J. (2004). Origin, structure, and role of background EEG activity. Part 1. Analytic amplitude. Clinical Neurophysiology, 115(9), 2077-2088.

Gao, R., Peterson, E. J., \& Voytek, B. (2017). Inferring synaptic excitation/inhibition balance from field potentials. NeuroImage, 158, 70-78.

Gárate-Pérez, M. F., Méndez, A., Bahamondes, C., Sanhueza, C., Guzmán, F., Reyes-Parada, M., Sotomayor-Zárate, R., \& Renard, G. M. (2021). Vasopressin in the lateral septum decreases conditioned place preference to amphetamine and nucleus accumbens dopamine release. Addiction Biology, 26(1), e12851.

González-Villar, A. J., Samartin-Veiga, N., Arias, M., \& Carrillo-de-la-Peña, M. T. (2017). Increased neural noise and impaired brain synchronization in fibromyalgia patients during cognitive interference. Scientific Reports, 7(1), 5841.

Jonsson, S., Morud, J., Stomberg, R., Ericson, M., \& Söderpalm, B. (2017). Involvement of lateral septum in alcohol's dopamine-elevating effect in the rat. Addiction Biology, 22(1), 93-102.

Koob, G. F., \& Volkow, N. D. (2016). Neurobiology of addiction: A neurocircuitry analysis. The Lancet, Psychiatry, 3(8), 760-773.

Kosko, B., \& Mitaim, S. (2003). Stochastic resonance in noisy threshold neurons. Neural Networks, 16, 755-761.

McDonnell, M. D., \& Ward, L. M. (2011). The benefits of noise in neural systems: Bridging theory and experiment. Nature Reviews, Neuroscience, 12(7), 415-426.

Miller, K. J., Sorensen, L. B., Ojemann, J. G., \& den Nijs, M. (2009). Power-law scaling in the brain surface electric potential. PLoS Computational Biology, 5(12), e1000609.

Miller, P., \& Katz, D. B. (2010). Stochastic transitions between neural states in taste processing and decision-making. The Journal of Neuroscience, 30(7), 2559-2570.

Molina, V., Bachiller, A., Suazo, V., Lubeiro, A., Poza, J., \& Hornero, R. (2016) Noise power associated with decreased task-induced variability of brain electrical activity in schizophrenia. European Archives of Psychiatry and Clinical Neuroscience, 266(1), 55-61.

Molina, J. L., Voytek, B., Thomas, M. L., Joshi, Y. B., Bhakta, S. G., Talledo, J. A., Swerdlow, N. R., \& Light, G. A. (2020). Memantine effects on electroencephalographic measures of putative excitatory/inhibitory balance in schizophrenia. Biological Psychiatry, Cognitive Neuroscience and Neuroimaging, 5(6), 562-568.

Muthukumaraswamy, S. D., \& Liley, D. T. (2018). 1/f electrophysiological spectra in resting and drug-induced states can be explained by the dynamics of multiple oscillatory relaxation processes. NeuroImage, 179, 582-595.

Osinski, B. L., Kim, A., Xiao, W., Mehta, N. M., \& Kay, L. M. (2018). Pharmacological manipulation of the olfactory bulb modulates beta oscillations: Testing model predictions. Journal of Neurophysiology, 120(3), 1090-1106.

Paxinos, G., \& Watson, C. (2006). The rat brain in stereotaxic coordinates. Academic Press, San Diego.

Pertermann, M., Bluschke, A., Roessner, V., \& Beste, C. (2019). The modulation of neural noise underlies the effectiveness of methylphenidate treatment in attention-deficithyperactivity disorder. Biological Psychiatry, Cognitive Neuroscience and Neuroimaging, 4(8), 743-750.
Pertermann, M., Mückschel, M., Adelhöfer, N., Ziemssen, T., \& Beste, C. (2019). On the interrelation of $1 / \mathrm{f}$ neural noise and norepinephrine system activity during motor response inhibition. Journal of Neurophysiology, 121(5), 1633-1643.

Porjesz, B., \& Begleiter, H. (2003). Alcoholism and human electrophysiology. Alcohol Research and Health, 27(2), 153-160.

Rangaswamy, M., \& Porjesz, B. (2014). Understanding alcohol use disorders with neuroelectrophysiology. Handbook of Clinical Neurology, 125, 383-414.

Ryabinin, A. E., Yoneyama, N., Tanchuck, M. A., Mark, G. P., \& Finn, D. A. (2008) Urocortin 1 microinjection into the mouse lateral septum regulates the acquisition and expression of alcohol consumption. Neuroscience, 151(3), 780-790.

Sartor, G. C., \& Aston-Jones, G. S. (2012). A septal-hypothalamic pathway drives orexin neurons, which is necessary for conditioned cocaine preference. The Journal of Neuroscience, 32(13), 4623-4631.

Schomburg, E. W., Anastassiou, C. A., Buzsáki, G., \& Koch, C. (2012). The spiking component of oscillatory extracellular potentials in the rat hippocampus. The Journal of Neuroscience, 32(34), 11798-11811.

Shannon, C. E. (1948). A mathematical theory of communication. The Bell System Technical Journal, 27(3), 379-423.

Stock, A. K., Pertermann, M., Mückschel, M., \& Beste, C. (2020). High-dose ethanol intoxication decreases $1 /$ f neural noise or scale-free neural activity in the resting state. Addiction Biology, 25(6), e12818.

Veerakumar, A., Tiruvadi, V., Howell, B., Waters, A. C., Crowell, A. L., Voytek, B., Riva-Posse, P., Denison, L., Rajendra, J. K., Edwards, J. A., Bijanki, K. R., Choi, K. S., \& Mayberg, H. S. (2019). Field potential 1/f activity in the subcallosal cingulate region as a candidate signal for monitoring deep brain stimulation for treatment-resistant depression. Journal of Neurophysiology, 122(3), 1023-1035.

Voytek, B., \& Knight, R. T. (2015). Dynamic network communication as a unifying neural basis for cognition, development, aging, and disease. Biological Psychiatry, 77(12), 1089-1097.

Voytek, B., Kramer, M. A., Case, J., Lepage, K. Q., Tempesta, Z. R., Knight, R. T. \& Gazzaley, A. (2015). Age-related changes in 1/f neural electrophysiological noise. The Journal of Neuroscience : the official journal of the Society for Neuroscience, 35(38), 13257-13265.

Wen, H., \& Liu, Z. (2015). Separating fractal and oscillatory components in the power spectrum of neurophysiological signal. Purdue University Research Repository, Purdue.

Wen, H., \& Liu, Z. (2016). Separating fractal and oscillatory components in the power spectrum of neurophysiological signal. Brain Topography, 29(1), 13-26.

Womelsdorf, T., Schoffelen, J. M., Oostenveld, R., Singer, W., Desimone, R., Engel, A. K., \& Fries, P. (2007). Modulation of neuronal interactions through neuronal synchronization. Science, 316(5831), 1609-1612.

Yamamoto, Y., \& Hughson, R. L. (1993). Extracting fractal components from time series. Physica D: Nonlinear Phenomena, 68(2), 250-264. 